\title{
Antimicrobial and Anti-Biofilm Activities of Citrus sinensis and Moringa oleifera Against the Pathogenic Pseudomonas aeruginosa and Staphylococcus aureus
}

\author{
Mohammad Zubair ${ }^{1}$ \\ 1. Medical Microbiology, University of Tabuk, Tabuk, SAU \\ Corresponding author: Mohammad Zubair,mohammad_zubair@yahoo.co.in
}

\begin{abstract}
Context

The plant Moringa oleifera Lam (Moringaceae), generally termed as drumstick tree, and Citrus sinensis Linn (Rutaceae) fruit have the ability to treat multiple human infections. A biofilm is none other than a complicated microbial community whose nature is greatly resistant to antimicrobial elements. The development of biofilms in abiotic and biotic surfaces has a connection with higher levels of mortality and morbidity. Along with that, it is regarded as a vital element of bacterial pathogenicity.
\end{abstract}

\section{Aim}

The present study evaluated the inhibitory effect and anti-biofilm activity of Moringa oleifera ( $M$. oleifera) and Citrus sinensis (C. sinensis) extracts against those of pathogenic Pseudomonas aeruginosa ( $P$. aeruginosa) and Staphylococcus aureus (S. aureus).

\section{Materials and methods}

Two plant materials were collected from the local market of Tabuk city and two human pathogenic microbial strains were used in the study: S. aureus and P. aeruginosa. Further, a series of morphological, physiological, and conventional biochemical tests were performed to identify the selected microorganisms. In addition to this, the study conducted the following tests: antibiotic sensitivity test, extended-spectrum $\beta$-lactamase (ES $\beta L$ ), and methicillin-resistant Staphylococcus aureus (MRSA) production, biofilm formation in 96-well microtiter plates, minimum inhibitory concentration (MIC) determination, the effect of sub-MICs of $C$. sinensis extract and M. oleifera extract on the viability of test bacteria, and finally, measurement of the inhibition of biofilm.

Review began $12 / 10 / 2020$ Review ended 12/21/2020 Published 12/28/2020

\section{() Copyright 2020}

Zubair. This is an open access article distributed under the terms of the Creative Commons Attribution License CC-BY 4.0., which permits unrestricted use, distribution, and reproduction in any medium, provided the original author and source are credited.

\section{Results}

A remarkable result of the research is that the peel extract of C. sinensis and the flesh extract of M. oleifera efficiently inhibited biofilm formation by the addition of sub-inhibitory concentrations of $(1 / 16 \mathrm{x}$ MIC $-1 / 2 \mathrm{x}$ MIC) MRSA and ESBL, respectively. P. aeruginosa shows high resistance to piperacillin (85.0\%). Similarly, the resistance of MRSA was also high (65\%) against gentamycin and amikacin antibiotics. Regarding ESBL, 12 (60\%) isolates showed confirmed positive and $45 \%$ of $S$. aureus showed MRSA activity. On observing the 12 ESBL-positive P. aeruginosa, it was found that five strains (PS1, PS4, PS6, PS8, and PS11) have formed strong biofilm, methicillin-resistant $S$. aureus while four strains showed strong biofilm activity (SA2, SA4, SA5, and SA8). The MIC of $C$. sinensis extract and M. oleifera extract against strong biofilm producers had a range of $50-2000 \mu \mathrm{g} / \mathrm{ml}$ concentration after overnight incubation. The study results revealed that the antibiofilm activity comparatively showed the extract of $M$. oleifera was better than $C$. sinensis against the mixed culture (PS1+SA8, PS6+SA2, and PS8+SA4). Hence, it is recommended to use M. oleifera as an option to monitor the development of microbial biofilms or as a model for looking for better medicines.

\section{Conclusion}

The presence of antimicrobial activity found in $M$. oleifera and C. sinensis extracts offers convincing evidence of their likely action as antimicrobial metabolites against the studied microorganism. Anti-biofilm assay findings have shown that $M$. oleifera and $C$. sinensis extracts have effectively blocked MRSA and ESBL development in the biofilm matrix.

Categories: Infectious Disease, Environmental Health, Epidemiology/Public Health Keywords: moringa oleifera, citrus sinensis extracts, s. aureus, pseudomonas species, tabuk city, es $\beta 1$ and mrsa production, biofilm formation 


\section{Introduction}

Antibiotics are being utilized for treating infections, in both animals and humans all over the world. Along with the therapeutic usage in animals, antibiotics are generally added in small quantities to animal feeds like prophylaxis and for the purpose of promoting growth [1]. A consistent increase has been noted in terms of microbial resistance to antimicrobials. In addition to that, a decreasing ability is found in the antimicrobials that are available for the purpose of treating general infections.

Antimicrobial resistance (AMR) is a vital risk when it comes to the health and welfare of the people as well as animals, which ends up with a great effect in terms of food security. Anyway, there is a dilemma all across the globe regarding the reduction of new therapeutic elements, which helps in treating different kinds of diseases that affect both animals as well as humans. There are chances for society to go through a postantibiotic period with present antibiotics getting ineffective slowly because of resistance. This contains big threats in terms of health and national security as well; for instance, bioterrorism and pandemics [2]. The estimation taken at the present time discloses a death toll of about 700,000 people annually. This is mainly because of the antibiotic resistance and a forecast that by 2050, there are higher chances for about 10 million people to be at threat, provided no measures are taken in stopping the drift of rising AMR [3]. In addition to that, it's presumed that people who live in developing nations that are subjected to mortality and morbidity due to the impact of infectious diseases shall be the ones who would be deadly affected by this condition.

The formation of biofilm is one among the strategies of resistance by several pathogens that indeed makes them even more complicated as compared to the platonic counterparts [4]. A biofilm is none other than a complicated matrix of microorganism communities that consist of proteins, polysaccharides, and other organic elements, wherein cells get fixed together, forming powerful attachments to abiotic and biotic surfaces [5]. Biofilms allow microbes that bind to a surface in order to persist despite harsh states like antimicrobial agents and host defenses [6]. Thus, the formation of biofilm is one of the indirect sources of action through which bacteria act resistant in terms of antibiotics and this is where even resistance genes get transferred among biofilm micro-community members [7]. P. aeruginosa and S. aureus are two, vital, opportunistic-natured pathogens all over the world, which cause nosocomial and community-acquired infections. Biofilm-related infections are caused by $P$. aeruginos $a$ and S. aureus, and methicillin-resistant $S$. aureus (MRSA) has evolved to be a pathogen (clinically appropriate) due to its nature of acting resistant toward antibiotics along with the potential of forming biofilms as examined by Alamri et al. [8]. Nearly $60 \%$ of microbial infections involve biofilms whilst $2 / 3$ rds of bacterial infections in humans are formed due to the biofilms [9].

M. oleifera Lam (Moringaceae) is a tree that has a fast-growing nature, and it is called drumstick tree or horseradish tree. A 4-( $\beta$-d-Glucopyranosyl-1 $\rightarrow 4-\alpha-1-$ rhamnopyranosyloxy) - benzyl thiocarboxamide, which are separated from the seed has displayed the potential activity of antimicrobials as stated by Oluduro et al. [10]. In the traditional aspect, the report says that when equal parts of $C$. sinensis Linn (Rutaceae) fruit rind (orange peel) and M. oleifera roots are blended, it is beneficial for the bowels as stated by Gholap et al. [11]. Therefore, this work was planned to study the inhibitory effect and anti-biofilm activity of $M$. oleifera and $C$. sinensis extracts against the most common clinical isolates (P. aeruginosa and S. aureus).

\section{Materials And Methods}

\section{Plant samples}

Two plant materials were collected on the basis of traditional medicinal history from the local market of Tabuk city (Table 1).

\begin{tabular}{|c|c|c|c|c|}
\hline $\begin{array}{l}\text { Botanical } \\
\text { Name }\end{array}$ & Common name & Family name & $\begin{array}{l}\text { Part } \\
\text { used }\end{array}$ & Ethnomedicinal use \\
\hline $\begin{array}{l}\text { Citrus } \\
\text { sinensis }\end{array}$ & Egyptian malta & Rutaceae & Peel & Treatment of cold, anorexia, and cough \\
\hline $\begin{array}{l}\text { Moringa } \\
\text { oleifera }\end{array}$ & $\begin{array}{l}\text { Horseradish tree, Radish tree, } \\
\text { Drumstick tree }\end{array}$ & Moringaceae & Flesh & $\begin{array}{l}\text { Curing of fever, infections in the ear, reduction of blood } \\
\text { sugar and pressure }\end{array}$ \\
\hline
\end{tabular}

\section{Collection of a bacterial sample}

Forty bacterial samples ( 20 S. aureus and 20 P. aeruginosa) were used in the study. To be doubly sure, basic biochemical tests were performed. The antibiotic assay was performed using the Kirby Bauer disc diffusion method following the Clinical and Laboratory Standards Institute (CLSI) guideline. 


\section{Extraction of test samples (a) C. sinensis (Egyptian malta), (b) $M$. oleifera}

The methodology of powder preparation of C. sinensis and M. oleifera was adopted from previously published reports [12]. The details are presented in the supplementary file.

\section{Antibiotic sensitivity testing of $P$. aeruginosa. and $S$. aureus}

Antibiotic sensitivity was performed on Mueller-Hinton agar by the Kirby-Bauer method. Amikacin (30 $\mu \mathrm{g})$, ceftazidime $(30 \mu \mathrm{g})$, cefepime $(30 \mu \mathrm{g})$, levofloxacin $(5 \mu \mathrm{g})$, tobramycin $(10 \mu \mathrm{g})$, piperacillin $(100 \mu \mathrm{g})$,

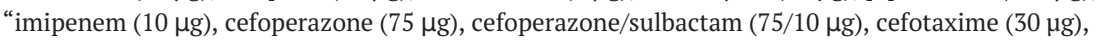
cefotaxime/clavulanic acid (30/10 $\mu \mathrm{g})$, piperacillin/tazobactam (100/10 $\mu \mathrm{g})$," cefepime clavulanic acid (30/10 $\mu g)$, sparfloxacin $(5 \mu \mathrm{g})$, tobramycin $(10 \mu \mathrm{g})$, "erythromycin (15 $\mu \mathrm{g})$, gentamicin $(10 \mu \mathrm{g})$, oxacillin $(1 \mu \mathrm{g})$, ciprofloxacin $(5 \mu \mathrm{g})$, cefoxitin (30 $\mu \mathrm{g})$, and vancomycin $(30 \mu \mathrm{g})$," were used in this study (Hi-Media Labs, Mumbai, India]. Interpretation of results as suggested by the manufacturer's recommendation (Hi-Media Labs).

\section{ES $\beta L$ and MRSA detection among isolated strains}

ES $\beta L$ producers were detected by using ceftazidime and cefotaxime alone and in combination with clavulanic acid $(10 \mathrm{mg})$, as recommended by the Clinical and Laboratory Standards Institute (CLSI) guidelines. For MRSA detection, the swab (sterile) was dipped in the $S$. aureus suspension ( $0.5 \mathrm{McFarland}$ ) and plated onto Mueller Hinton agar (MHA). Oxacillin discs $(1 \mu \mathrm{g})$ were used with an overnight incubation at $30^{\circ} \mathrm{C}$. When the zone of inhibition was $\leqslant 14 \mathrm{~mm}$ in diameter, it was considered resistant to oxacillin. The control strains used in this study were "E. coli ATCC 25922 (non-ESBL-producer), K. pneumoniae 700603 (ESBL-producer), S. aureus (ATCC 25923).”

\section{Biofilm formation in 96-well microtiter plates}

Biofilm formation was examined by the quantitative determination of biofilm formation in 96-well flatbottom plates by Coffey and Anderson [13]. For each clinical strain tested, biofilm assays were performed in triplicate and the mean biofilm absorbance value was determined. The biofilms formed were classified as weak (OD590 0.1 to $\leqslant 0.400)$, moderate (OD590 >0.400), and strong (OD590 >0.800).

\section{Minimum inhibitory concentration (MIC) determination}

The MIC of C. sinensis extract and M. oleifera extract against resistant biofilm-forming strains of $P$. aeruginosa and $S$. aureus was estimated using the standard micro-broth dilution method recommended CLSI guidelines at $37^{\circ} \mathrm{C}$ at $600 \mathrm{~nm}$ after $24 \mathrm{~h}$ incubation.

\section{Effect of $C$. sinensis extract and $M$. oleifera extract on mono and mixed-species biofilms}

In inhibition assays, bacteria inoculated in microtitre plates were treated with 1/18-1/2 x MICs of C. sinensis extract and M. oleifera extract and incubated at $37^{\circ} \mathrm{C}$ for $48 \mathrm{~h}$. The inhibition of biofilm was measured as described in the previous section. The mixed biofilm formation was quantified as described by Zhang et al. [14].

\section{Statistical analysis}

Data represented in the manuscripts were mean value, experiments were performed in triplicate, and tests were analyzed using the student's t-test.

\section{Results}

Overall, 40 bacterial strains ( 20 S. aureus and 20 P. aeruginosa.) were collected from various hospitals in the city of Tabuk, Kingdom of Saudi Arabia. The collected bacteria were allowed to get subjected to the process of drug resistance observation (tabulated results are shown in Table 2). 


\section{Cureus}

\begin{tabular}{|c|c|c|c|c|}
\hline \multirow{2}{*}{ a) Resistance pattern } & \multicolumn{2}{|l|}{ P. aeruginosa } & \multirow{2}{*}{ b) Resistance pattern } & S. aureus \\
\hline & \multicolumn{2}{|l|}{$\mathrm{N}=20$} & & $\mathrm{~N}=20$ \\
\hline Amikacin & \multicolumn{2}{|l|}{$15(75.0)$} & Amikacin & $13(65.0)$ \\
\hline Ceftazidins & \multicolumn{2}{|l|}{$9(45.0)$} & Erythromycin & $10(10.0)$ \\
\hline Cefepime & \multicolumn{2}{|l|}{$14(70.0)$} & Ciprofloxacın & $9(45.0)$ \\
\hline Levofloxacin & \multicolumn{2}{|l|}{$15(75.0)$} & Gentamycin & $13(65.0)$ \\
\hline Spartloxacin & \multicolumn{2}{|l|}{$15(75.0)$} & Levofloxacin & $10(50.0)$ \\
\hline Tobramycin & \multicolumn{2}{|l|}{ 14(70.0) } & Oxacillin & $9(45.0)$ \\
\hline Piperacillin & \multicolumn{2}{|l|}{$17(85.0)$} & Vancomycin & $0(0)$ \\
\hline \multicolumn{5}{|l|}{ c) ESBL pattern } \\
\hline \multicolumn{5}{|l|}{ Prelımınary test } \\
\hline Ceftazidime & \multicolumn{3}{|c|}{$14(70.0)$} & \\
\hline Cefotaxime & \multicolumn{3}{|c|}{$15(75.0)$} & \\
\hline \multicolumn{5}{|l|}{ Contirmatory lest } \\
\hline Ceftazıdıme/Ceftazıdime Clavulanuc acid & & $12(60.0)$ & & \\
\hline Cefotaxime/Cefotaxime Clavulanuc acid & & $12(60.0)$ & & \\
\hline \multicolumn{5}{|l|}{ d) MRSA results } \\
\hline Oxacillin & & $9(45.0)$ & & \\
\hline
\end{tabular}

TABLE 2: Antibiotic resistance pattern: a) P. aeruginosa ; b) S. aureus; c) ESBL pattern; d) MRSA pattern

P. aeruginosa. Pseudomonas aeruginosa; S. aureus. Staphylococcus aureus; ESBL: extended-spectrum beta-lactamase; MRSA: methicillin-resistant Staphylococcus aureus

\section{Antibiotic resistance pattern}

P. aeruginosa shows high resistance to piperacillin (85.0\%) followed by amikacin, levofloxacin, and sparfloxacin, which show $75 \%$, respectively. Similarly, the resistance of S. aureus was also high (65\%) against gentamycin and amikacin antibiotics, respectively. Regarding ESBL, 12 (60\%) isolates showed confirmed positive and $45 \%$ of S. aureus showed MRSA activity. For the antibiofilm activity of the C. sinensis extract and the M. oleifera extract, MRSA and ES $\beta$ L-positive P. aeruginosa were selected for biofilm activity.

\section{Biofilm activity of $\boldsymbol{P}$ aeruginosa and $S$. aureus}

The mono species biofilm formation among MRSA and ES $\beta$ L-positive $P$. aeruginosa were classified as strong, moderate, weak, and negative (Table 3). On observing the 12 ESBL-positive P. aeruginosa strains, it is found that five strains (PS1, PS4, PS6, PS8, and PS11) have formed strong biofilms, two strains as moderate (PS2 and PS9), and two as weak biofilms (PS7 and PS10) (Figure 1A). In the case of methicillin-resistant S. aureus, four strains show strong biofilm activity (SA2, SA4, SA5, and SA8), whereas two strains (SA1, SA6) were moderate formers and SA9 was a weak biofilm producer (Figure 1B). For the antibiofilm activity of C. sinensis extract and M. oleifera extract, only the strong biofilm-positive methicillin-resistant $S$. aureus (SA2, SA4, SA5, and SA8) and P. aeruginosa (PS1, PS2, PS6, PS12, and PS18) were selected for further experiments. 


\section{Cureus}

\begin{tabular}{|c|c|c|c|c|}
\hline & \multicolumn{3}{|c|}{ Biofilm producers } & \multirow[b]{2}{*}{ Negative $\mathrm{n}(\%)$} \\
\hline & Strong n (\%) & Moderate n (\%) & Weak n (\%) & \\
\hline P. aeruginosa $(n=12)$ & 5 & 2 & 3 & 2 \\
\hline S. aureus ( $n=9$ ) & 4 & 2 & 1 & 2 \\
\hline
\end{tabular}

TABLE 3: Classification of $P$. aeruginosa and $S$. aureus for biofilm activity as strong, moderate, and weak (data are $\mathbf{n}(\%)$ unless otherwise indicated)

P. aeruginosa. Pseudomonas aeruginosa; S. aureus. Staphylococcus aureus
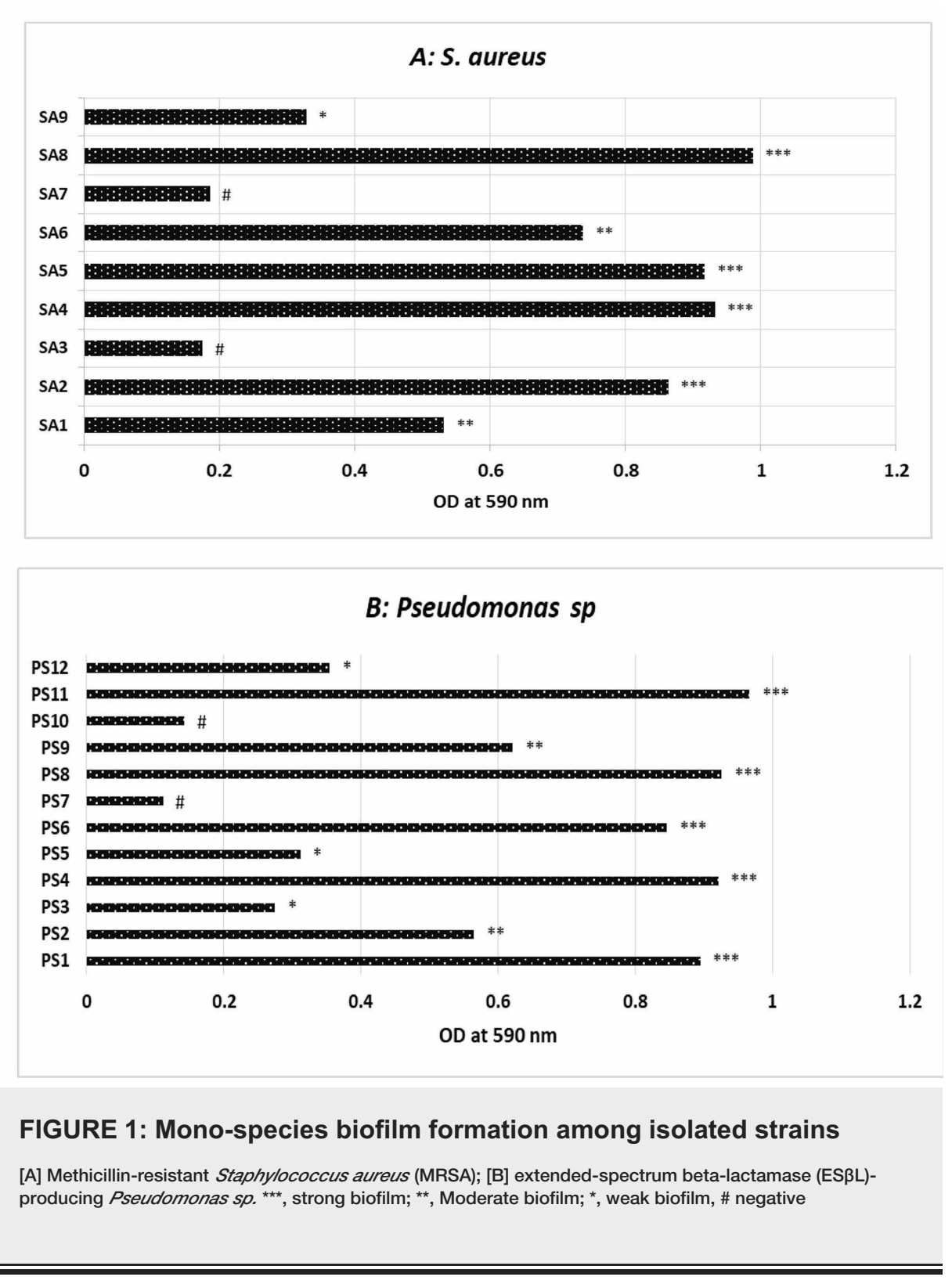

MICs of C. sinensis extract and M. oleifera extract

The MIC of $C$. sinensis extract and $M$. oleifera extract against strong biofilm producers with a range from 50$2000 \mu \mathrm{g} / \mathrm{ml}$ concentrations after overnight incubation are depicted in Table 4. 


\section{Cureus}

\begin{tabular}{|c|c|c|c|c|c|c|c|c|c|c|}
\hline \multirow{2}{*}{$\begin{array}{l}\text { Strains } \\
\text { ESBL }\end{array}$} & \multirow[t]{2}{*}{$\begin{array}{l}\text { MIC of Citrus sinensis } \\
\text { extract }(\mu \mathrm{g} / \mathrm{ml})\end{array}$} & \multicolumn{4}{|c|}{$\begin{array}{l}\text { Sub-MIC of Citrus sinensis extract } \\
(\mu \mathrm{g} / \mathrm{ml})^{a}\end{array}$} & \multirow[t]{2}{*}{$\begin{array}{l}\text { MIC of Moringa oleifera } \\
\text { extract }(\mu \mathrm{g} / \mathrm{ml})\end{array}$} & \multicolumn{4}{|c|}{$\begin{array}{l}\text { Sub-MIC of Moringa oleifera extract } \\
(\mu \mathrm{g} / \mathrm{ml})^{\mathrm{b}}\end{array}$} \\
\hline & & 1/16xMMC & 1/8xMIC & 1/4xMIC & 1/2xMIC & & 1/16xMIC & 1/8xMIC & 1/4xMIC & $1 / 2 \times M I C$ \\
\hline PS1 & 200 & 12.5 & 25 & 50 & 100 & 1200 & 75 & 150 & 300 & 600 \\
\hline PS4 & 300 & 18.7 & 37.5 & 75 & 150 & 1000 & 62.5 & 125 & 250 & 500 \\
\hline PS6 & 100 & 6.25 & 12.5 & 25 & 50 & 800 & 50 & 100 & 200 & 400 \\
\hline PS8 & 400 & 25 & 50 & 100 & 200 & 1600 & 100 & 200 & 400 & 800 \\
\hline PS11 & 350 & 21.8 & 43.7 & 87.5 & 175 & 1000 & 62.5 & 125 & 250 & 500 \\
\hline \multicolumn{11}{|l|}{ MRSA } \\
\hline SA2 & 100 & 6.25 & 12.5 & 25 & 50 & 800 & 50 & 100 & 200 & 400 \\
\hline SA4 & 400 & 25 & 50 & 100 & 200 & 1600 & 100 & 200 & 400 & 800 \\
\hline SA5 & 600 & 37.5 & 75 & 150 & 300 & 2000 & 125 & 250 & 500 & 1000 \\
\hline SA8 & 200 & 12.5 & 25 & 50 & 100 & 1200 & 75 & 150 & 300 & 600 \\
\hline
\end{tabular}

TABLE 4: MIC of Citrus sinensis extract and Moringa oleifera extract strong biofilm-forming ESBLproducing Pseudomonas and methicillin-resistant (MRSA) strains.

MIC: minimum inhibitory concentration; ESBL: extended-spectrum beta-lactamase; MRSA: methicillin-resistant Staphylococcus aureus

The sub-lethal concentrations dose were selected for biofilm inhibition assay (Table 4). The adding of extracts of C. sinensis and M. oleifera at respective 1/2 x MIC at the beginning of the growth showed no change in the growth of strong biofilm-positive MRSA (SA2, SA4, SA5, and SA8) (Figure 2A). A similar pattern was also observed for ESBL-positive, strong biofilm-producing P. aeruginosa (PS1, PS2, PS6, PS12, and PS18) for C. sinensis and M. oleifera at respective 1/2 x MIC (Figure 2B). To avoid the reduction of biofilm formation activity, this sub-MIC was performed. 


\section{Cureus}
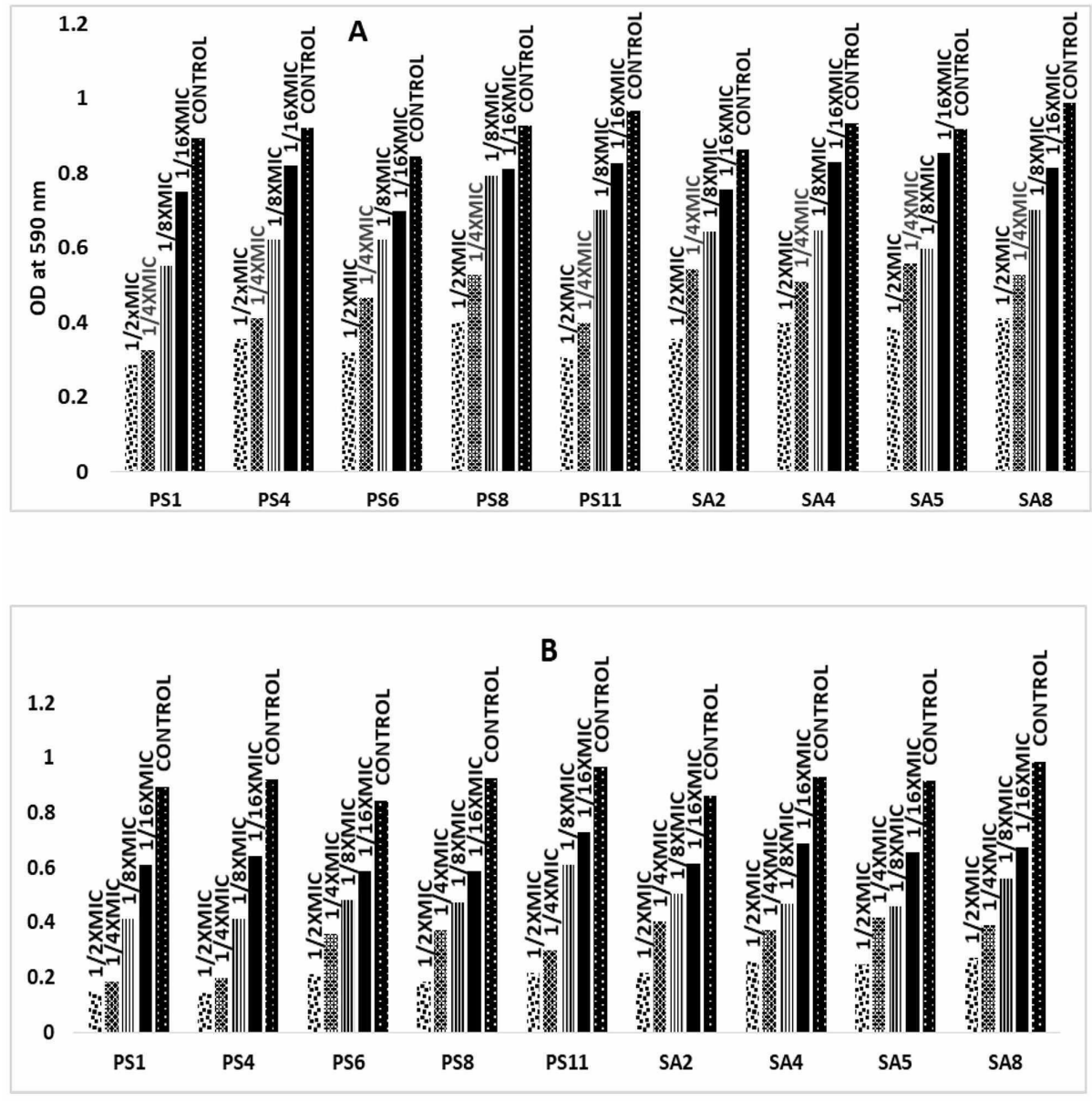

FIGURE 2: Inhibition of biofilm formation by sub-inhibitory concentrations of $[\mathrm{A}] \mathrm{C}$. sinensis extract; $[\mathrm{B}] \mathrm{M}$. oleifera extract. The data represent the mean values of three independent experiments.

C. sinensis. Citrus sinensis; M. oleifera. Moringa oleifera

Inhibition of mono-culture biofilm by $C$. sinensis extract and $M$. oleifera extract

Figure $2 \mathrm{~A}$ bar graphs indicate the inhibition of biofilm formation by sub-inhibitory concentrations of $C$. sinensis extract (1/16 x MIC - 1/2 x MIC). The 14\%-68\% reduction of biofilm formation by ESBL-producing $P$. aeruginosa (PS11); $16 \%-68 \%$ by PS1; $17 \%-62 \%$ by PS6; $61 \%-10 \%$ by PS4; and $12 \%-56 \%$ by PS8 as compared to control. The $12 \%-59 \%$ of MRSA (SA2); followed by SA8 (17\%-58 \%); SA4 (11\%-57\%), and SA5 (6\%-57\%) (Table 5, panel A). 


\section{Cureus}

\begin{tabular}{|c|c|c|c|c|c|c|c|c|}
\hline \multicolumn{9}{|l|}{ A: Monoculture } \\
\hline \multirow{3}{*}{ Isolate Name } & \multicolumn{4}{|c|}{ C. sinensis extract } & \multicolumn{4}{|c|}{ M. oleifera extract } \\
\hline & \multicolumn{4}{|c|}{ Reduction percentage at } & \multicolumn{4}{|c|}{ Reduction percentage at } \\
\hline & \multicolumn{2}{|l|}{ 1/16xMIC } & \multicolumn{2}{|l|}{ 1/2xMIC } & \multicolumn{2}{|l|}{ 1/16xMIC } & \multicolumn{2}{|l|}{ 1/2xMIC } \\
\hline PS1 & \multicolumn{2}{|l|}{$16 \%$} & \multicolumn{2}{|l|}{$68 \%$} & \multicolumn{2}{|l|}{$31 \%$} & \multicolumn{2}{|l|}{$86 \%$} \\
\hline PS4 & \multicolumn{2}{|l|}{$10 \%$} & \multicolumn{2}{|l|}{$61 \%$} & \multicolumn{2}{|l|}{$30 \%$} & \multicolumn{2}{|l|}{$84 \%$} \\
\hline PS6 & \multicolumn{2}{|l|}{$17 \%$} & \multicolumn{2}{|l|}{$62 \%$} & \multicolumn{2}{|l|}{$30 \%$} & \multicolumn{2}{|l|}{$75 \%$} \\
\hline PS8 & \multicolumn{2}{|l|}{$12 \%$} & \multicolumn{2}{|l|}{$56 \%$} & \multicolumn{2}{|l|}{$36 \%$} & \multicolumn{2}{|l|}{$80 \%$} \\
\hline PS11 & \multicolumn{2}{|l|}{$14 \%$} & \multicolumn{2}{|l|}{$68 \%$} & \multicolumn{2}{|l|}{$24 \%$} & \multicolumn{2}{|l|}{$77 \%$} \\
\hline SA2 & \multicolumn{2}{|l|}{$12 \%$} & \multicolumn{2}{|l|}{$59 \%$} & \multicolumn{2}{|l|}{$28 \%$} & \multicolumn{2}{|l|}{$75 \%$} \\
\hline SA4 & \multicolumn{2}{|l|}{$11 \%$} & $57 \%$ & & $25 \%$ & & $72 \%$ & \\
\hline SA5 & $6 \%$ & & $57 \%$ & & $28 \%$ & & $73 \%$ & \\
\hline SA8 & $17 \%$ & & $58 \%$ & & $31 \%$ & & $72 \%$ & \\
\hline B: Mixed Cultu & & & & & & & & \\
\hline & C. sinensis & ract & & & M. oleifera & ract & & \\
\hline Isolate Name & Reduction & centage at & & & Reduction & centage at & & \\
\hline & 1/16xMIC & 1/8xMIC & 1/4xMIC & 1/2xMIC & 1/16xMIC & 1/8xMIC & 1/4xMIC & 1/2xMIC \\
\hline PS1+SA8 & $14 \%$ & $32 \%$ & $46 \%$ & $46 \%$ & $47 \%$ & $51 \%$ & $68 \%$ & $74 \%$ \\
\hline PS6+SA2 & $15 \%$ & $23 \%$ & $40 \%$ & $62 \%$ & $54 \%$ & $60 \%$ & $71 \%$ & $76 \%$ \\
\hline PS8+SA4 & $10 \%$ & $18 \%$ & $43 \%$ & $56 \%$ & $46 \%$ & $54 \%$ & $67 \%$ & $73 \%$ \\
\hline
\end{tabular}

TABLE 5: Reduction percentage of sub-inhibitory concentrations (for monoculture at 1/16xMIC1/2xMIC; mixed culture at 1/16xMIC-1/8xMIC-1/4xMIC-1/2xMIC) of C. sinensis extract and M. oleifera extract against biofilm formation

C. sinensis. Citrus sinensis, M. oleifera: Moringa oleifera; MIC: minimum inhibitory concentration

The bar graphs in Figure $2 B$ graphs indicate the inhibition of biofilm formation by sub-inhibitory concentrations of $M$. oleifera extract (1/16 x MIC - 1/2 x MIC). The 31\%-86\% reduction of biofilm formation by ESBL-producing $P$. aeruginosa (PS1); 30\%-84\% by PS4; 36\%-80\% by PS8; $24 \%-77 \%$ by PS11; and 30\%-75\% by PS6 as compared to control. Then $28 \%-75 \%$ by MRSA (SA2); followed by SA5 (28\%-73\%); SA4 (25\%-72\%) and SA8 (31\%-72\%) (Table 5).

\section{Inhibition of mixed biofilm}

Figure 3 (panels A-B) illustrates the comparative antibiofilm activity of C. sinensis extract and $M$. oleifera extract against the mixed culture (PS1+SA8, PS6+SA2, and PS8+SA4). The overall antibiofilm activity of $M$. oleifera was reported better as compared with C. sinensis (Table 5 panel B) when compared with controls at 1/16xMIC-1/8xMIC-1/4xMIC-1/2xMIC, respectively. 


\section{Cureus}
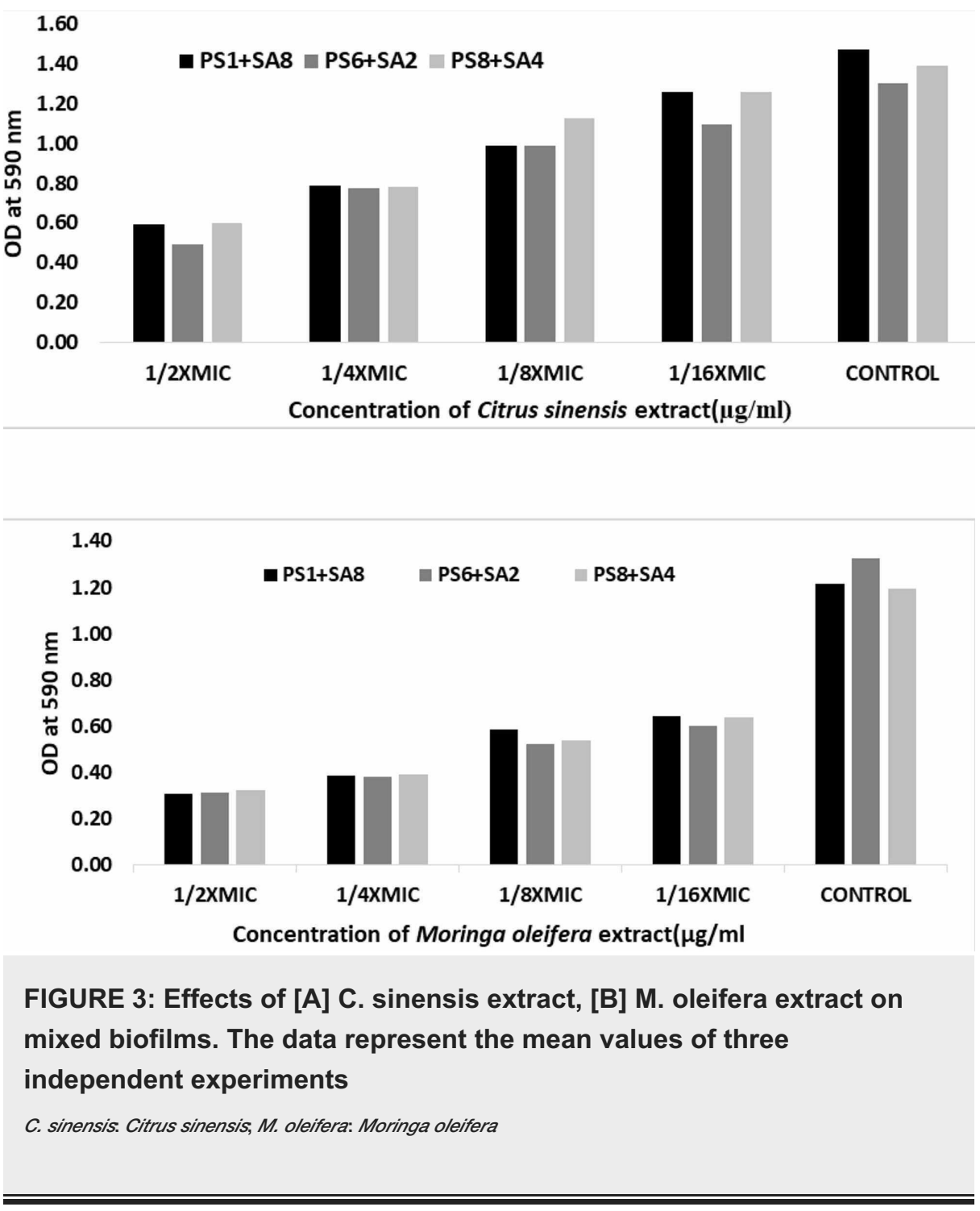

\section{Discussion}

Biofilms are being identified as crucial in human disease, and the number of infections associated with biofilms is growing [6]. S. aureus, for example, has been shown to be among the most difficult pathogens implicated in a number of infections [15] such as indwelling medical device (IMD) infections associated with it. It has been noted that it is getting impossible to remove Staphylococcus spp biofilm infections when most of the drugs on the market have to be used as combination therapies [16]. Likewise, P. aeruginosa has arisen within immune-compromised individuals as one of the chief reasons for nosocomial infections [17].

The ESBL detection test showed $60 \%$ of $P$. aeruginosa were ES $\beta$ L producers and the maximum of them showed high resistance to piperacillin (85.0\%), which resembles the findings outlined by Harris et al. [18]. Piperacillin-tazobactam is commonly used in seriously ill patients to treat $P$. aeruginosa infections. The new U.S. medications are ceftazidime-avibactam and ceftolozane-tazobactam. Food and Drug Administration (FDA)-approved combinations of cephalosporin- $\beta$-lactamase inhibitors to treat Gram-negative bacilliinduced infections, including P. aeruginosa [19]. Similarly, the resistance of MRSA was also high (65\%) against gentamycin and amikacin antibiotics, respectively, and $45 \%$ of $S$. aureus shows MRSA activity. The maximum isolates of S. aureus (84.5\%) were comparable to other studies in terms of resistance to gentamycin [20].

The mono species biofilm formation among MRSA S. aureus and ES $\beta$ L-positive $P$. aeruginosa were classified as strong, moderate, weak, and negative. On observing the 12 ESBL-positive Pseudomonas strains, it was found that five strains (PS1, PS4, PS6, PS8, and PS11) formed strong biofilms. In the case of methicillinresistant $S$. aureus, four strains show strong biofilm activity (SA2, SA4, SA5, and SA8). Strong biofilm (referring to +++ ) showed a significantly higher likelihood of tolerance or resistance to antibiotics that will probably result in therapeutic failure in MRSA infections [21]. There is no prevailing opinion to date on the categorization of $S$. aureus isolates predicated on their biofilm-forming capability. The concept of a strong, medium, weak, and non-biofilm producer consequently varies widely between studies [22]. 
The MIC of $C$. sinensis extract and $M$. oleifera extract against strong biofilm producers is in the range of 50$2000 \mu \mathrm{g} / \mathrm{ml}$ concentrations after overnight incubation. Similar to our study, the study by Ahmad and Aqil [23] showed the MICs measured from 64 to $1024 \mu \mathrm{g} / \mathrm{ml}$ for cefotaxime, cefuroxime, ampicillin, and penicillin.

Adding the extracts of $C$. sinensis and $M$. oleifera at respective $1 / 2 \times$ MIC at the beginning of the growth showed no change in the growth of strong biofilm-positive methicillin-resistant $S$. aureus (SA2, SA4, SA5, and SA8). A similar pattern was also observed for ESBL-positive, strong biofilm-producing $P$. aeruginosa. (PS1, PS2, PS6, PS12, and PS18) for C. sinensis and M. oleifera at respective $1 / 2 \times$ MIC. The formation of biofilms depends on several variables, including the environment, nutrient availability, geographical origin, specimen forms, and properties of surface adhesion and genetic composition of the species [24]. The data may have been influenced by these variables and led to the high prevalence found in the current research. It is not, however, understood as to how these variables are concerned.

Biofilms include MRSA and ESBL with a defensive barrier to withstand antibiotic therapy. A remarkable result of the research is that the peel extract of $C$. sinensis and a flesh extract of $M$. oleifera efficiently inhibited the biofilm-formation by the addition of sub-inhibitory concentrations of (1/16 x MIC - $1 / 2 \times$ MIC) MRSA and ESBL, respectively. This indicates the acquired effect was dose-dependent. The better biofilm reduction by ESBL-producing $P$. aeruginos $a$ is observed at higher concentrations of $C$. sinensis extracts at 14\%-68\% in PS11. Similar findings have been observed by Abraham et al. [25], who documented that methanolic caper extraction substantially inhibited biofilm formation and extracellular polymeric substance (EPS) development in Proteus mirabilis, P. aeruginosa, Serratia marcescens, and E. coli. Likewise, the better biofilm reduction of MRSA is observed at higher concentrations at 12\%-59\% in SA2. A similar pattern was also observed in $M$. oleifera extracts (1/16 x MIC - 1/2 x MIC). The 31\%-86\% \% reduction of biofilm formation by ESBL-producing Pseudomonas (PS1); 30\%-84 \% by PS4; 36\%-80\% by PS8; $24 \%-77 \%$ by PS11; and 30\%-75\% by PS6 as compared to controls. The $28 \%-75 \%$ by MRSA (SA2); followed by SA5 (28\%-73 \%), SA4 (25\%-72\%), and SA8 (31\%-72\%). The existence of previously identified flavonoids, such as quercetin, kaempferol, naringenin, and apigenin, which are capable of reducing biofilm synthesis because they can suppress the activity of the autoinducer- 2 responsible for cell-to-cell contact, can explain the inhibition of biofilm formation [26]. The inhibitory activity demonstrated by $C$. sinensis and $M$. oleifera could be derived from its ability to synthesize metabolites that can prevent the formation of biofilms. This result, however, needs to be further investigated through sophisticated qualitative and quantitative studies. A literature survey reveals that the antibiofilm activity by sub-inhibitory concentrations of $C$. sinensis and M. oleifera against MRSA and ESBL is scanty. Though, the antibiofilm perspective of flavonoids extracted from M. oleifera seed coat in contradiction to $S$. aureus is stated from India [27].

Our findings concerning the function of antibiofilms agree with previous work carried out in other terrestrial plant species from various parts of the world. Research from India, for example, reported that the Vetiveria zizanioides root extract showed an inhibition reduction in MRSA biofilm formation [28]. Similarly, another study conducted in Brazil found that Piper regnellii's dichloromethane extract weakens biofilm formation [29].

The mono and mixed-species biofilm activity was quantified against M. oleifera and $C$. sinensis. The findings of the antibiofilm activity comparatively showed that the extract of $M$. oleifera was reported better as compared with $C$. sinensis against the mixed culture (PS1+SA8, PS6+SA2, and PS8+SA4). Hence, it is recommended to use $M$. oleifera as an option to monitor the development of microbial biofilms or as a model for looking for better medicines. Clinically, biofilm infections are significant, whereas bacteria show recalcitrance to antimicrobial compounds. Large concentrations of antimicrobials may be required to eliminate biofilm producers. Owing to the possibility of toxicity and the associated side effects, this might not always be feasible in vivo, however, low-concentration combination therapies can, therefore, be effective in eradicating staphylococcal biofilm-related infections, including those induced by MRSA [30]. For the selection of a suitable antimicrobial agent, the early screening and detection of biofilm producers followed by their antimicrobial susceptibility tests is essential.

\section{Conclusions}

In the present study, according to my knowledge, this was the first report of the antimicrobial and antibiofilm activity of $M$. oleifera and $C$. sinensis in the Kingdom of Saudi Arabia. This study also provides better efficacy of $M$. oleifera over $C$. sinensis in its antibiofilm activity in both mono and mixed culture in vitro experiments. Further studies are required on the molecular mechanism involved in controlling the resistance by $M$. oleifera. It could, therefore, be concluded that new groups of anti-biotic leads will be provided by the bioassay-guided fractionation and purification of M. oleifera and C. sinensis.

\section{Appendices}

\section{Preparation of C. sinensis peel powder}

C. sinensis (oranges) was procured from the local fruit market and any extraneous materials were first by washing. The orange peel was resized into $1 \mathrm{x} 1$ inch and dried at $40^{\circ} \mathrm{C}$ till its moisture content reduced to $<5 \%$. It was finely ground into powder. The 60 gram of fine powder of peel powder was dissolved in $160 \mathrm{ml}$ of 
absolute ethanol at room temperature for three successive days. Using Whatman paper, the supernatant was filtered, and residues were used for a second and third extraction. For three days, dissolved parts were filtered and stored in a glass bottle. After the third extraction, the filtrates were then evaporated under reduced pressure at $50^{\circ} \mathrm{C}$ using a rotary evaporator to yield the crude extract [12].

Percentage Yield $(\%)=[$ Dry weight of the extract $/$ Dry weight of plant material $] \times 100$

The crude extract was collected in a vial for further use.

\section{Preparation of $M$. oleifera (fruit flesh) powder}

M. oleifera was procured from the local fruit market and any materials were first by washing. The flesh was resized into $1 \mathrm{x} 1 \mathrm{inch}$ and dried at $40^{\circ} \mathrm{C}$ till its moisture content reduced to $<5 \%$. It was finely grounded into a powder. The 60 gram of fine powder of peel powder was dissolved in $160 \mathrm{ml}$ of absolute ethanol at room temperature for three successive days. Using Whatman paper, the supernatant was filtered and residues were used for a second and third extraction. For three days, dissolved parts were filtered and stored in a glass bottle. After the third extraction, the filtrates were then evaporated under reduced pressure at $50^{\circ} \mathrm{C}$ using a rotary evaporator to yield the crude extract [12].

Percentage Yield $(\%)=[$ Dry weight of the extract $/$ Dry weight of plant material $] \times 100$

The crude extract was collected in a vial for further use.

\section{Effect of $C$. sinensis extract and $M$. oleifera extract on mono and mixed-species biofilm}

In brief, each strain (S. aureus and $P$. aeruginosa) was grown overnight at $37^{\circ} \mathrm{C}$ in tryptic soy broth (TSB) and diluted to 1 x $106 \mathrm{CFU} / \mathrm{ml}$ in the TSB. Equal numbers (1:1) of each bacterium were mixed together and the mixed bacterial suspension $(100 \mu \mathrm{l})$ was added to each well of the polystyrene 96 -well tissue-culture plates. Each well was filled with $100 \mu \mathrm{l}$ of fresh TSB containing different concentrations of $C$. sinensis extract (1/16$1 / 2 \times$ MIC). Negative control wells contained TSB only, and wells with no additives were used as positive controls. After incubation for $48 \mathrm{~h}$ at $37^{\circ} \mathrm{C}$, plates were gently washed with $1 \mathrm{X}$ phosphate-buffered saline (PBS; pH 7.4) and stained with $100 \mu$ l of $0.1 \%$ crystal violet (CV) (Sigma-Aldrich, St. Louis, MO) for 30 min at room temperature. Excess CV was removed by washing, and biofilm was quantified by measuring the corresponding OD590 $\mathrm{nm}$ of the supernatant following the solubilization of CV in $95 \%$ ethanol. The experiment was repeated with $M$. oleifera extract effect on mono and mixed biofilm study [14].

\section{Additional Information \\ Disclosures}

Human subjects: All authors have confirmed that this study did not involve human participants or tissue. Animal subjects: All authors have confirmed that this study did not involve animal subjects or tissue. Conflicts of interest: In compliance with the ICMJE uniform disclosure form, all authors declare the following: Payment/services info: All authors have declared that no financial support was received from any organization for the submitted work. Financial relationships: All authors have declared that they have no financial relationships at present or within the previous three years with any organizations that might have an interest in the submitted work. Other relationships: All authors have declared that there are no other relationships or activities that could appear to have influenced the submitted work.

\section{Acknowledgements}

The author is very thankful to all the associated personnel in any reference that contributed to/for this research.

\section{References}

1. Hashemi SR, Davoodi H: Herbal plants and their derivatives as growth and health promoters in animal nutrition. Vet Res Commun. 2011, 35:169-180. 10.1007/s11259-010-9458-2

2. Lowrence RC, Ramakrishnan A, Sundaramoorthy NS, et al.: Norfloxacin salts of carboxylic acids curtail planktonic and biofilm mode of growth in ESKAPE pathogens. J Appl Microbiol. 2018, 124:408-422. 10.1111/jam.13651

3. Yelin I, Kishony R: SnapShot: antibiotic resistance. Cell. 2018, 172:1136-1136. 10.1016/j.cell.2018.02.018

4. de la Fuente-Núñez C, Korolik V, Bainset M, et al.: Inhibition of bacterial biofilm formation and swarming motility by a small synthetic cationic peptide. Antimicrob Agents Chemother. 2012, 56:2696-2704 10.1128/AAC.00064-12

5. Bazargani MM, Rohloff J: Antibiofilm activity of essential oils and plant extracts against Staphylococcus aureus and Escherichia coli biofilms. Food Control. 2016, 61:156-164. 10.1016/j.foodcont.2015.09.036

6. Jamal M, Ahmad W, Andleeb S, et al.: Bacterial biofilm and associated infections. J Chinese Med Assoc. 2018, 81:7-11. 10.1016/i.jcma.2017.07.012 
7. Lebeaux D, Ghigo JM, Beloin C: Biofilm-related infections: bridging the gap between clinical management and fundamental aspects of recalcitrance toward antibiotics. Microbiol Mol Biol Rev. 2014, 78:510-543. 10.1128/MMBR.00013-14

8. Alamri AM, Alsultan AA, Ansari MA, Alnimr AM: Biofilm-formation in clonally unrelated multidrugresistant Acinetobacter baumannii isolates. Pathogens. 2015, 9:630. 10.3390/pathogens 9080630

9. de la Fuente-Núñez C, Cardoso HM, de Souza Cândido E, et al.: Synthetic antibiofilm peptides . Biochim Biophys Acta Biomembr. 2016, 1858:1061-1069. 10.1016/j.bbamem.2015.12.015

10. Oluduro OA, Aderiye BI, Connolly JD, Akintayo ET, Famurewa O: Characterization and antimicrobial activity of 4-( $\beta$-d-glucopyranosyl-1 $\rightarrow 4-\alpha$-l-rhamnopyranosyloxy)-benzyl thiocarboxamide; a novel bioactive compound from Moringa oleifera seed extract. Folia Microbiol. 2010, 55:422-426. 10.1007/s12223-0100071-0

11. Gholap PA, Nirmal SA, Pattan SR, Pal SC, Mandal SC: Potential of Moringa oleifera root and Citrus sinensis fruit rind extracts in the treatment of ulcerative colitis in mice. Pharm Biol. 2012, 50:1297-1302. 10.3109/13880209.2012.674142

12. Masad AA, Bashiti TA, Mosleh F, Abu Madi UM: Antibacterial and antibiofilm activity of selected plant extract against some human pathogenic microorganism. Pak J Nutr. 2019, 18:1014-1020. 10.3923/pjn.2019.1014.1020

13. Coffey BM, Anderson GG: Biofilm Formation in the 96-Well Microtiter Plate. Pseudomonas Methods and Protocols. Filloux A, Ramos JL (ed): Humana Press, New York; 2014. 631-641. 10.1007/978-1-4939-0473$0 \_48$

14. Zhang QQ, Ye KP, Wang HH, Xiao HM, Xu XL, Zhou GH: Inhibition of biofilm formation of Pseudomonas aeruginosa by an acylated homoserine lactones-containing culture extract. LWT - Food Sci Technol. 2014, 57:230-235. 10.1016/j.lwt.2013.12.022

15. Taylor TA, Unakal CG: Staphylococcus aureus. StatPearls Publishing, Treasure Island (FL); 2020.

16. Khatoon Z, McTiernan CD, Suuronen EJ, Mah TF, Alarcon EI: Bacterial biofilm formation on implantable devices and approaches to its treatment and prevention. Heliyon. 2018, 4:E01067. 10.1016/j.heliyon.2018.e01067

17. Pachori p, Gothalwal R, Gandhi P: Emergence of antibiotic resistance Pseudomonas aeruginosa in intensive care unit; a critical review. Genes Dis. 2019, 6:109-119. 10.1016/j.gendis.2019.04.001

18. Harris AD, Perencevich E, Roghmann MC, Morris G, Kaye KS, Johnson JA: Risk factors for piperacillintazobactam-resistant Pseudomonas aeruginosa among hospitalized patients. Antimicrob Agents Chemother. 2002, 46:854-858. 10.1128/AAC.46.3.854-858.2002

19. Allergan, Avycaz (ceftazidime-avibactam) for injection for intravenous use. California, United States, 2016 . (2016). https://www.accessdata.fda.gov/drugsatfda_docs/label/2017/206494s003lbl.pdf.

20. Khosravi AD, Jenabi A, Montazeri EA: Distribution of genes encoding resistance to aminoglycoside modifying enzymes in methicillin-resistant Staphylococcus aureus (MRSA) strains. Kaohsiung J Med Sci. 2017, 33:587-593.

21. 21] Manandhar S, Singh A, Varma A, Pandey S, Shrivastava N: Biofilm producing clinical Staphylococcus aureus isolates augmented prevalence of antibiotic resistant cases in tertiary care hospitals of Nepal. Front Microbiol. 2018, 9:2749. 10.3389/fmicb.2018.02749

22. Crémet L, Corved S, Batard S, et al.: Comparison of three methods to study biofilm formation by clinical strains of Escherichia coli. Diagn Microbiol Infect Dis. 2013, 75:252-255. 10.1016/j.diagmicrobio.2012.11.019

23. Ahmad I, Aqil F: In vitro efficacy of bioactive extracts of 15 medicinal plants against ES $\beta$ L-producing multidrug-resistant enteric bacteria. Microbiol Res. 2007, 162:264-275. 10.1016/j.micres.2006.06.010

24. Kokare CR, Chakraborty S, Khopade SN, Mahadik KR: Biofilm: importance and applications. Indian J Biotechnol. 2009, 8:159-168.

25. Sánchez E, Rivas Morales C, Castillo S, Leos-Rivas C, García-Becerra L, Ortiz Martínez DM: Antibacterial and antibiofilm activity of methanolic plant extracts against nosocomial microorganisms: evidence-based complement. Altern Med. 2016, 2016:1572697. 10.1155/2016/1572697

26. Vikram A, Jayaprakasha GK, Jesudhasan PR, Pillai SD, Patil BS: Suppression of bacterial cell-cell signalling, biofilm formation and type III secretion system by citrus flavonoids. J Appl Microbiol. 2010, 109:515-527.

27. Onsare JG, Arora DS: Antibiofilm potential of flavonoids extracted from Moringa oleifera seed coat against Staphylococcus aureus, Pseudomonas aeruginosa and Candida albicans. J Appl Microbiol. 2015, 118:313325 .

28. Kannappan A, Gowrishankar S, Srinivasan R, Pandian SK, Ravi AV: Antibiofilm activity of Vetiveria zizanioides root extract against methicillin-resistant Staphylococcus aureus. Microb Pathog. 2017, 110:313324. 10.1016/j.micpath.2017.07.016

29. Brambilla LZS, Endo EH, Cortez DAG, Dias Filho BP: Anti-biofilm activity against Staphylococcus aureus MRSA and MSSA of neolignans and extract of Piper regnellii. Rev Bras Farmacogn. 2017, 27:112-117. 10.1016/j.bjp.2016.08.008

30. Wu WS, Chen CC, Chuang YC, et al.: Efficacy of combination oral antimicrobial agents against biofilmembedded methicillin-resistant Staphylococcus aureus. J Microbiol Immunol Infect. 2013, 46:89-95. 10.1016/j.jmii.2012.03.009 